\title{
Early Progressive Atrioventricular Block After Amplatzer Septal Occluder Closure of a Large Atrial Septal Defect
}

\author{
Derek A. Williams, DO*; Allison Welch, PA-C; Yoshio Ootaki, MD, PhD \\ Brenner Children's Hospital, Wake Forest University Baptist Medical Center, Winston-Salem, North Carolina, United States
}

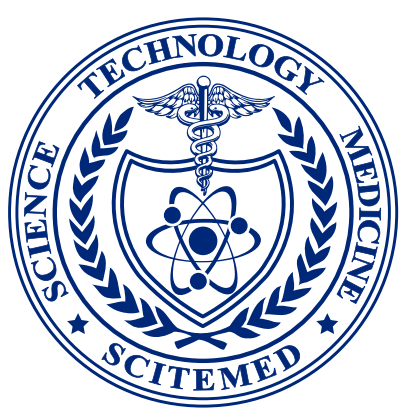

\begin{abstract}
Authors describe a 4-year-old boy with a moderate secundum atrial septal defect who developed rapidly progressive heart block after device closure. After surgical removal and patch closure he had gradual return to sinus rhythm. This report highlights the continued needed vigilance of heart block after atrial septal defect device closure.
\end{abstract}

\section{INTRODUCTION}

Risks for transcatheter device closure of secundum atrial septal defects (ASD) are low with the majority known to be thromboembolic events, erosion, nickel hypersensitivity, migraines, valvular damage, atrial arrhythmias, endocarditis, and conduction abnormalities [1-5]. Some degree of atrioventricular block is the most common form of conduction abnormality occurring between 1-7\% [6-8]. Transcatheter closure of the ASD has been in practice for more than 40 years. As such, in the current era, it has become the preferred method for closure with great success. Despite the low complication rates, the search continues to fully understand which patients are best suited for device closure vs surgical closure and/or what procedural changes should be made to further lessen the complications rates when a moderate or large ASD with deficient rims exists. We describe a case of progressive atrioventricular(AV) block in the first 12 hours after Amplatzer Septal Occluder (ASO) closure of a secundum ASD.

\section{CASE REPORT}

A 4-year-old born at 35-week gestation with a weight of $2.5 \mathrm{~kg}$ and a maternal history of cocaine and crack abuse was seen for the first time by pediatric cardiology for a newly heard heart murmur at 2 years of age. He was in the 3 and 10 th percentile for weight and height. His care givers were concerned he tired easily with play and had had one episode of unresponsiveness at the kitchen table. His electrocardiogram (ECG) revealed normal sinus rhythm and rate for age as well as prominent right ventricular forces (Figure 1). His echocardiogram (ECHO) revealed a moderate to large secundum ASD with right atrial and ventricular dilation. He was followed annually for two years when the decision was made to close his ASD. His weight was $13.8 \mathrm{~kg}$ (7th percentile). His transthoracic ECHO showed the ASD to measure $15 \mathrm{~mm}$ in diameter. Intervention was performed under general anesthesia. His trans-esophageal ECHO showed his septal length to be $31 \mathrm{~mm}$ with the ASD measuring $18 \mathrm{~mm} \times 20$ $\mathrm{mm}$ (Figure 2A). The retro-aortic rim was deficient measuring $5 \mathrm{~mm}$ (Figure 2B) and the inferior vena cava (IVC) rim was thought to be $11 \mathrm{~mm}$ but floppy. Stop flow performed with a $30 \mathrm{~mm}$ PTS $®$ sizing balloon (B.Braun, Bethelem, PA. ) showed a $22 \mathrm{~mm}$ waist. During slow balloon inflation sinus bradycardia was seen on the monitor. The balloon was advanced slightly into the left atrium and re-inflated with the same result. The ASD was closed using a $22 \mathrm{~mm}$ ASO. The left atrial disk was deployed in the right upper pulmonary vein to catch the retro-aortic rim. With the device fully deployed but still attached to the delivery cable first degree AV block was seen and confirmed with a rate of 100 beats per minute (bpm) (Figure 3A). The device released with good alignment with the plane of the atrial septum with transesophageal echocardiography (TEE) showing possible trivial residual left to right shunting near the IVC rim (Figure 2C). The patient recovered well and was placed on telemetry overnight with first degree AV block. Approximately, 12 hours later telemetry revealed progression to $2^{\text {nd }}$ degree AV block (2:1) with heart rates in the 60-bpm range (Figure 3B). This was confirmed with ECG. The patient remained comfortable and stable in bed. Within the hour, intermittent short periods of $3^{\text {rd }}$ degree (complete) heart block were seen. Therefore, the decision was made to surgically remove the device and patch closure the ASD. The operation took place approximately 18 hours post device placement and the device was seen overlapping the coronary sinus and well within the Triangle of Koch where the AV node resides (Figure 4). The device was safely removed and patch closure of the ASD was uneventful (Figure 5). The patient remained in $3^{\text {rd }}$ degree heart block with a rate of $40 \mathrm{bpm}$ the first two days after surgery so was atrial pacing paced using temporary epicardial pacing wires. He returned to $2^{\text {nd }}$, then $1^{\text {st }}$ degree heart block. Sinus rhythm returned post-op day 7 . He was in sinus rhythm at the time of discharge home. He remained in sinus rhythm at his 1- and 4-week follow-up.

\section{DISCUSSION}

Secundum ASD was the first congenital defect attempted to be surgically corrected in the 1950's [9]. Therefore, it has a long history of procedural improvements and well-defined complications. Presently, the majority of secundum ASDs are closed percutaneous with great success [10]. The devices available for percutaneous closure have been refined since the 1970s with the ASO having the longest longevity of usage. A few other options are available in the United States and still more internationally. The ASO seems to be the device of choice for moderate to large secundum ASDs as the other devices presently available do not have as great success achieving closure. The ASO make up has been well described with self-expanding right and left atrial discs with a central waist which fills the ASD and polyester material which aids in ASD occlusion and tissue in-growth. It is stated to have a $98.5 \%$ closure rate at one-year follow-up [2]. Also, the potential adverse events are well known with heart block being covered under the more general heading of "arrhythmia" with an "arrhythmia" rate listed of only $0.5 \%$ during the pivotal study. Differing degrees of heart block have been sporadically described after ASO closure but mostly in the form of case studies or small number studies [4-7]. Some thought 


\section{CASE REPORT}

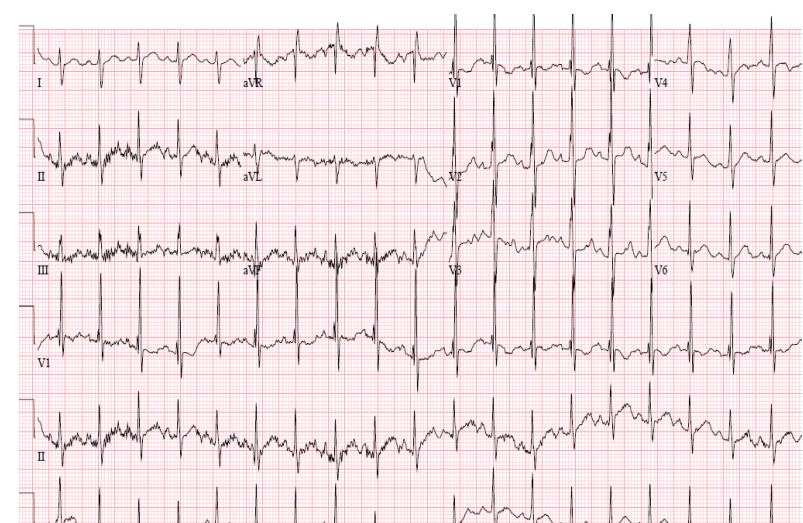

Figure 1. Pre-catheterization electrocardiogram.
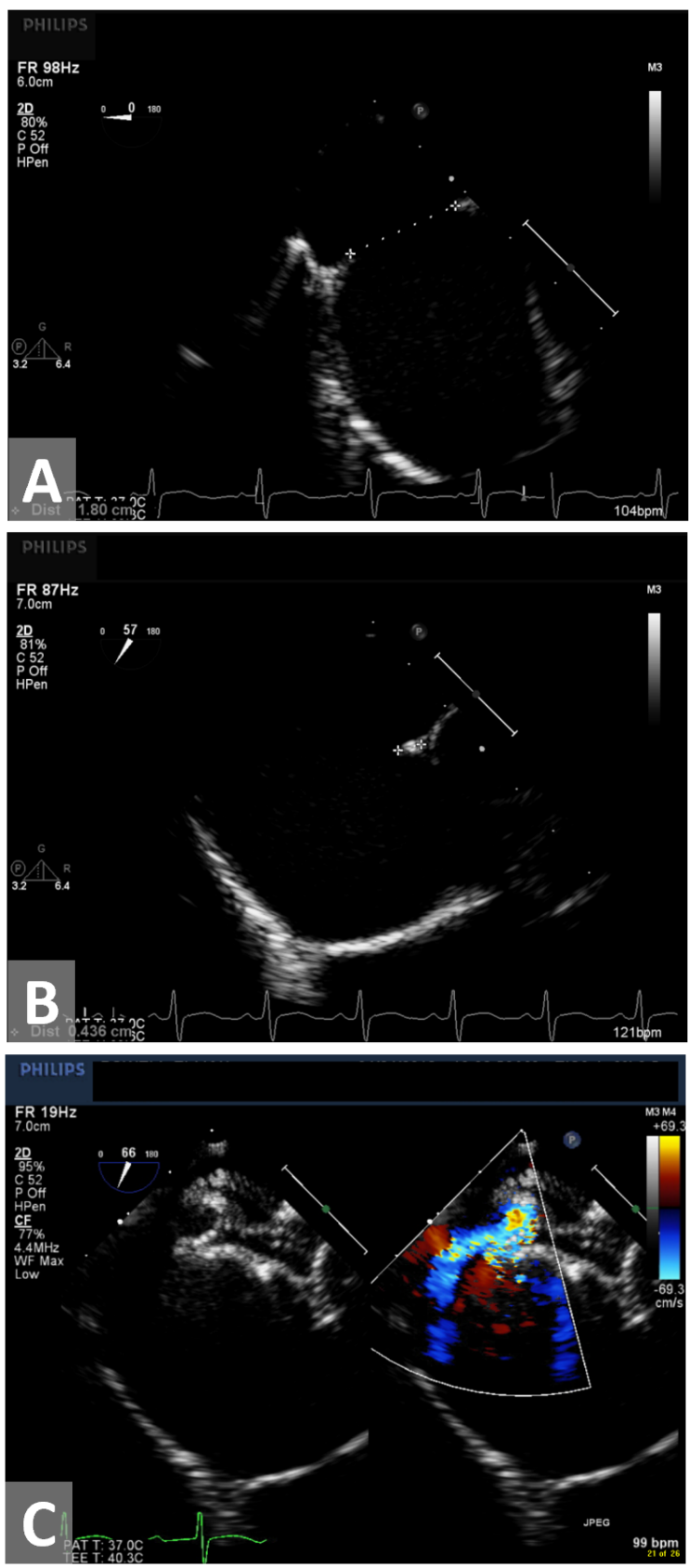

Figure 2. Transesphageal echocardiogram. (A) Diameter of secundum atria septal defects. (B) Deficient retro-aortic rim measuring $4.4 \mathrm{~mm}$. (C) $22 \mathrm{~mm} \mathrm{Am}$ platzer Septal Occluder deployed with trivial residual shunt near inferior vena cava rim.

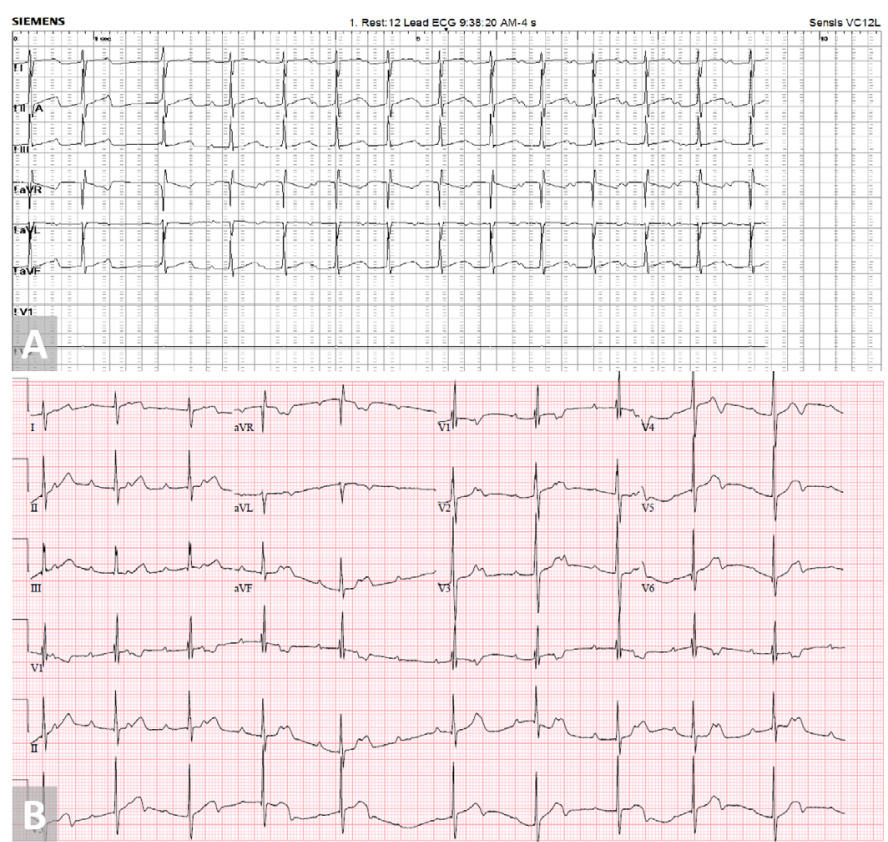

Figure 3. (A) First degree atrioventricular block seen in catheterization lab with Amplatzer Septal Occluder deployment. (B) $3^{\text {rd }}$ degree atrioventricular block seen 12 hours post Amplatzer Septal Occluder deployment.

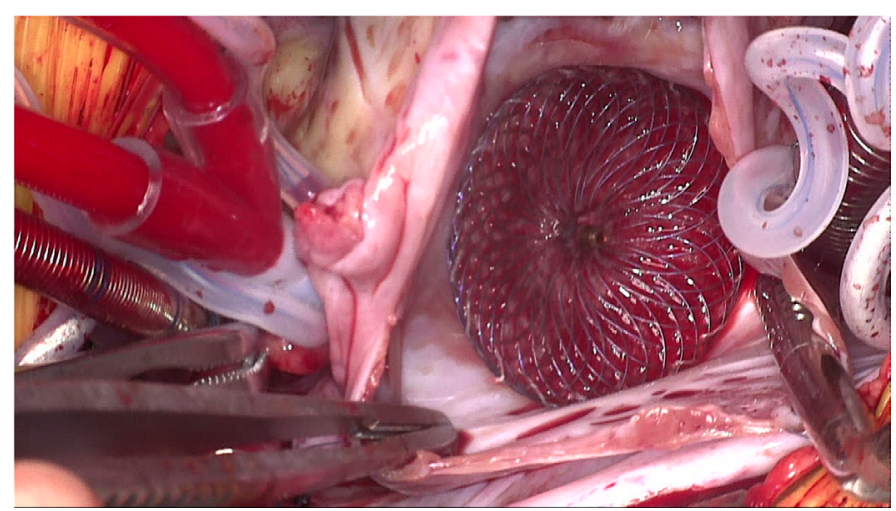

Figure 4. Amplatzer Septal Occluder seen overlapping the Coronary Sinus and Triangle of Koch (right atrial side).

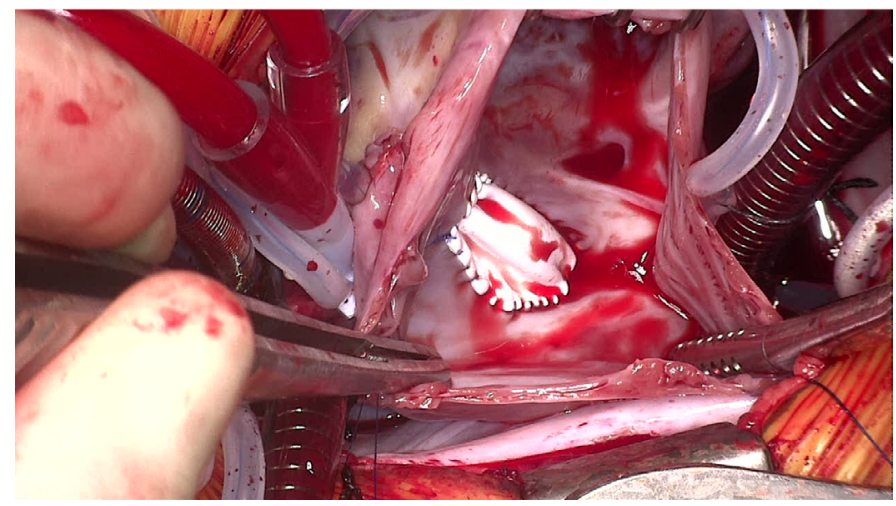

Figure 5. Patch closure of atrial septal defects.

has been given as to whether the ASO device design makes it susceptible to causing AV block but the Gore Septal Occluder (GSO ${ }^{\mathrm{TM}}$ )has reported as well [10]. Some studies have proposed an elevated ratio of ASO/atrial septal length and/or deficient aortic or posterior rim may be predictive of higher incidence of AV block [7,11]. Also, the decision to treat medically or remove the device is not well defined $[6,10,12,13]$. 
Our case was particularly interesting for several reasons. First, we noticed the IVC rim was difficult to measure accurately as it appeared floppy. This then could account for a deficient rim and this is where the small residual shunting was seen post device placement. Second, bradycardia occurred with the balloon inflation stop-flow technique despite repositioning of the balloon toward the left atrium. This is not a typical occurrence and may have signaled a higher likelihood of post device arrhythmia. Third, despite the device being fairly large for the patient's size, it had to be released from the right upper pulmonary vein in order to capture the small aortic rim. Additionally, we notice first degree heart block with deployment of the right atrial disc which persisted after device release despite release of the device tension. Also, our patient progressed to second degree heart block and then third degree in approximately 12 hours despite being clinically stable. Finally, sinus rhythm took several days post device removal to return.

As first degree heart block (PR interval prolongation) can be a normal finding on routine ECG, we did not believe it to be a contra-indication for ASO release in our patient thinking it would spontaneously resolve. The option always exists to not release the ASO from the delivery cable and remove if concerns exist. Additionally, the ASO has a good success rate for removal post release if complications arise. Also, for the first 8 or so hours post device placement, first degree heart block remained without progression. When 2:1 heart block occurs, there are three options. Treat with NSIADS and steroids which have worked in some cases thought to combat the AV nodal swelling $[6,10,12,13]$. Return to the catheterization lab, remove the device and either use a different device or wait until the patient grows larger and see what new devices are then available. The third option is surgical removal with patch closure. We chose this option as the appearance of third-degree heart block ensued. The in-situ surgical picture showed the device covering the coronary sinus and the right atrial disc well into the Triangle of Koch where the AV node resides. The ASD patch closure occupied much less space and relieved both. Since sinus rhythm returned, likely AV nodal swelling had occurred but what will remain a mystery is whether advance heart block would have remained if the device remained in place.

\section{CONCLUSION}

As complications of percutaneous ASD closure continue, although rare, defining which patient are most appropriate for this type of closure needs to be continually refined. We could suggest that based on our patient's device/ septal length ratio (0.73), there was a higher risk of AV block [8]. Additionally, if an adverse event such as arrhythmia or AV block with stop-flow balloon inflation and/or device deployment without release shows the same. Alternatively, we could suggest simply a low threshold for device removal given the low incidence of advanced heart block. Perhaps device design will continue to progress to where risk of AV block disappears. We would suggest given this case and the other sporadic cases published each institution performing device closure have pre-established plans about treatment when advance AV block occurs. As despite now more than 40 years of device improvements for secundum ASD closure, the risk AV block persists.

\section{ARTICLE INFORMATION}

*Correspondence: Derek A. Williams, DO, Brenner Children's Hospital, Wake Forest University Baptist Medical Center, Medical Center Boulevard, Winston-Salem, NC, 27157, USA. Email: dewillia@wakehealth.edu

Received: Mar. 19, 2019; Accepted: Aug. 02, 2019; Published: Sep. 09, 2019

\section{DOI: $10.24983 /$ scitemed.acri.2019.00118}

Ethics Approval and Consent to Participate: The study is in accordance with the ethical standards of the 1964 Helsinki declaration and its later amendments or comparable ethical standards.

Funding: The study did not receive any specific grant from funding agencies in the public, commercial, or not-for-profit sectors.

Conflict of Interest: The authors report no financial or other conflict of interest relevant to this article, which is the intellectual property of the authors.

Copyright $@ 2019$ The Authors. This is an open-access article distributed under the terms of the Creative Commons Attribution 4.0 International License (CC-BY).

\section{REFERENCES}

1. Abbott, Structure Heart Occluders, Important Saftey Imformation, Adverse Events. 2019. Available at: https://www.cardiovascular.abbott/us/ en/hcp/therapies/structural-heart/structural-intervention-occluders. html

2. U.S. Food and Drug Administration. The Amplatzer ${ }^{\circledR}$ Septal Occluder (ASO) and the Amplatzer Exchange System Premarket Approval 2001. Available at: https://www.accessdata.fda.gov/scripts/cdrh/cfdocs/cfp$\mathrm{ma} / \mathrm{pma}$.cfm?id=P000039

3. Fornell D. FDA Reports Serious Erosion Events With Amplatzer Septal Occluder. Diagnostic and Interventional Cardiology 2013. Available at: https:// www.dicardiology.com/article/fda-reports-serious-erosion-events-amplatzer-septal-occluder.

4. Jalal Z, Hascoet S, Bartuteau A, et al. Long-term complications after transcatheter atrial septal defect closure: a review of the medical literature. Can J Cardiol 2016;32(11):1315.e11-1315.e18.

5. Bartakian S, Fagan TE, Schaffer MS, Darst JR. Device closure of secundum atrial septal defects in children <15 kg: Complications rates and indications for referral. JACC Cardiovasc Interv 2012;5(11):1178-1184.

6. Hill SL, Berul Cl, Patel HT, et al. Early ECG abnormalities associated with transcatheter closure of atrial septal defects using the Amplatzer septal occluder. J Interv Card Electrophysiol 2000; 4(3):469-474.

7. Al-Anani SJ, Weber $\mathrm{H}$, Hijazi ZM. Atrioventricular block after transcatheter ASD closure using the Amplatzer Septal Occluder: risk factors and recommendations. Catheter Cardiovasc Interv 2010;75(5):767-772.

8. Wang Y, Hua Y, Li L, et al. Risk factors and prognosis of atrioventricular block after atrial septum closure using the Amplatzer device. Pediatr Cardiol 2014;35(5):550-555.

9. King TD, Mills NL, King NB. A history of ASD closure. Cardiac Interventions Today 2010;55-61.

10. Tadroa VX, Asgar AW. Transcatheter closure of atrial septal defects. Card Interv Today 2016;10:56-60.

11. Dittrich $S$, Sigler M, Priessmann H. Late Complete Atrioventricular Block After Closure of an Atrial Septal Defect With a Gore Septal Occluder (GSO ${ }^{\mathrm{TM}}$ ). Catheter Cardiovasc Interv 2016;87(5):945-950.

12. Jin M, Ding WH, Wang XF, et al. Value of the ratio of occluder versus atrial septal length for predicting arrhyhtmia occurrence after transcatheter closure in children with ostium secundum atrial septal defect. Chin Med J (Engl). 2015;128(12):1574-1578.

13. Rohit MK, Puri K, Vadivelu R. Reversible complete atrioventricular block after percutaneous ASD device closure in a child $<15 \mathrm{~kg}$. India Heart J 2014;66(3):366-369. 